\title{
Diabetic ketoacidosis without hyperglycemia as a complication of SGLT2 inhibitors treatment
}

\section{ABSTRACT}

SGLT2 inhibitors (gliflozins) are a new group of hypoglycemic drugs which have been available on the market for the past four years. They inhibit the reabsorption of glucose in the proximal renal tubules, reduce the renal glucose threshold and increase glycosuria, thereby lowering blood glucose levels. Clinical recommendations of the Polish Diabetes Association mention the possibility of using gliflozins both as monotherapy in patients with type 2 diabetes, as well as in combination with other hypoglycemic drugs, including insulin. Databases of adverse events of SGLT2 inhibitors indicate an increased risk of diabetic ketoacidosis without significant hyperglycemia. An unusual course of this form of acidosis makes the complication difficult to diagnose. This paper describes a possible pathogenic process of ketoacidosis without hyperglycemia. The fact that this complication was observed mainly in patients with prolonged type 2 diabetes (including patients treated with insulin) and in patients with type 1 diabetes who used the drug "off-label", implies that insulin deficiency may be a pathogenic factor in this disorder. Another important factor was insufficient carbohydrate intake associated with reduced food consumption due to illness or accompanying disorders. At the same time, in most cases, a decrease in insulin dose stemming from low blood glucose levels was

Address for correspondence:

lek. Monika Trojanowska-Grigoriew

Klinika Diabetologii i Chorób Wewnętrznych

Pomorskiego Uniwersytetu Medycznego w Szczecinie

e-mail: monikagrigoriew@gmail.com

Translation: GROY Translations

Clinical Diabetology 2016, 5, 2, 66-72

DOI: $10.5603 /$ DK.2016.0011

Received: 02.03.2016

Accepted: 04.04.2016 observed. The study highlighted the precautions and prevention methods for ketoacidosis without hyperglycemia in patients treated with SGLT2 inhibitors, as well as proper and rapid diagnosis of this complication. (Clin Diabet 2016; 5, 2: 66-72)

Key words: diabetes, SGLT2 inhibitors, diabetic ketoacidosis without hyperglycemia

\section{Introduction}

Diabetes is currently classified into the heterogeneous group of metabolic diseases characterized by hyperglycemia. It stems from a defect in insulin production and/or function. It is one of the most common chronic diseases in the modern society. Currently there are 415 million people with diabetes around the world. It is estimated that this number will reach around 642 million in 2040 . Over $90 \%$ of people with the disease have type 2 diabetes [1].

Despite an asymptomatic or oligosymptomatic course, type 2 diabetes is not a mild disease. Effects of longlasting diabetes include dysfunction, damage and insufficiency of multiple organs. Each year the disease causes 5 million deaths. Diabetes is also the main cause of myocardial infarctions, cerebral strokes, amputations, renal insufficiency and blindness. Diagnosed in middle age, it shortens the average lifespan by 5-10 years compared to the general population [2-4].

The main clinical challenge of modern diabetology is optimizing and increasing effectiveness of treatment for type 2 diabetes. Despite employing various classes of drugs directed at pathogenic mechanisms of diabetes, such as insulin resistance and dysfunctions in insulin secretion, the effects of treatment remain unsatisfactory. Recently, kidneys have become the target organ for 
one of the classes of drugs which reduce blood glucose level. Kidneys play a vital role in regulating glucose concentration by utilizing glucose for metabolic needs, gluconeogenesis, glucose filtration and reabsorption of approximately $180 \mathrm{~g}$ of glucose per day [5-7].

\section{Sodium-glucose cotransporter $\mathbf{2}$ inhibitors}

Sodium-dependent glucose transporters (SGLT) belong to the great family of transmembrane proteins and are responsible for intestinal glucose absorption and renal glucose reabsorption. So far, six isoforms of these cotransporters have been described, although most likely the most important functions among them are performed by two: SGLT1 and SGLT2 [5].

The SGLT1 protein is a glucose and galactose transport system and is characterized by high affinity and low capacity. It is mostly found in brush border cells in the small intestine and is responsible for absorbing glucose/galactose in food. This transporter can also be found in the S3 segment of nephron's proximal tubules, in the brain, in the skeletal muscles and the cardiac muscle, in the liver and in the lungs. The SGLT2 sodium-glucose cotransporter can be found almost exclusively only in the brush border of the $\mathrm{S} 1$ segment of nephron's proximal tubule [6]. It is characterized by low affinity, but high capacity in respect to glucose. SGLT2 is responsible for reabsorption of $90 \%$ of glucose in the primary filtrate. The remaining glucose is reabsorbed by SGLT1.

Historically, the first SGLT cotransporters inhibitor was phlorizin, discovered in 1835. It was isolated from apple tree bark. Due to its bitter taste, similar to that of willow tree or cinchona tree extracts, it was thought that it would have similar properties and was initially used as a medicine for fever and infectious diseases, including malaria. Fifty years later it was found that large amounts of phlorizin cause glycosuria [8]. Being a non-specific SGLT1 and SGLT2 inhibitor, phlorizin found no use in treatment of diabetes since it caused severe gastrointestinal symptoms linked to its impediment of intestinal glucose absorption due to it blocking SGLT1. Furthermore, it was poorly absorbed from the gastrointestinal tract.

Modern synthetic analogs of phlorizin have considerable selectivity in respect to SGLT2 and so do not cause any gastrointestinal side effects.

Currently, three SGLT2 inhibitors (so-called gliflozins) have been approved by the European Medicines Agency (EMA) for marketing in the European Union, and by the Food and Drug Administration (FDA) in the United States.

In Europe, dapagliflozin (Forxiga, AstraZeneca) [9] was the first to be introduced in November 2012. In
November 2013, canagliflozin (Invokana, Janssen) [10] was introduced. The youngest, empagliflozin, was approved in the European Union in May 2014 (Jardiance, Boehringer Ingelheim) [11].

In the United States, canagliflozin (Invokana, Janssen) [12] was the first to be introduced in March 2013. Dapagliflozin (Farxiga, AstraZeneca) was approved for marketing in January 2014 [13]. Empagliflozin (Jardiance, Boehringer Ingelheim) has been present on the American market since August 2014 [14].

Combination preparations containing SGLT2 inhibitors are also available. These are combinations of dapagliflozin with metformin (Xigduo, AstraZeneca), canagliflozin with metformin (Vokanamet, Janssen) and empagliflozin with metformin (Synjardy, Boehringer Ingelheim), available in both Europe and the United States. In the United States, a product which is a combination of empagliflozin and linagliptin (Glyxambi, Boehringer Ingelheim) has also been introduced.

Further drugs from the SGLT2 inhibitors category are in various phases of pre-registration research. In Japan, ipragliflozin, tofogliflozin and luseogliflozin are available.

All SGLT2 inhibitors can be used as monotherapy in patients with type 2 diabetes where diet and exercise alone do not provide satisfactory control of blood glucose level and metformin cannot be administered for various reasons. This class of drugs can be used in combination therapy with other hypoglycemic drugs, including insulin [15].

Hypoglycemic action of this class of drugs does not depend on preserved residual function of pancreatic cells. SGLT2 inhibitors, by impeding glucose reabsorption in renal proximal tubules, decrease renal glucose threshold and increase glycosuria [16-18]. The amount of glucose removed by the kidneys depends on the blood glucose concentration and glomerular filtration rate (GFR). Inhibiting SGLT2 effectively reduces glucose concentration in the serum, while retaining SGLT1 activity prevents hypoglycemia. An increased level of SGLT2 mRNA was found in the cells isolated from proximal tubules of patients with type 2 diabetes compared to healthy people [19]. However, SGLT2 inhibitors only block reabsorption of approximately $30-50 \%$ of glucose excreted into primary urine [20].

The benefits of using SGLT2 inhibitors are not limited only to improvement in the balance of carbohydrate metabolism parameters. An important advantage of these drugs is their contribution to body weight loss and reduction in blood pressure while being used [21-24].

Results of research on effectiveness and safety of empagliflozin were presented in October 2015 during 
an EASD meeting in Stockholm and published in New England Journal of Medicine. During the EMPA-REG trial, adding empagliflozin to the current standard treatment for patients with type 2 diabetes with high risk of cardiovascular events reduced the risk of those events, including death from cardiovascular causes, non-fatal myocardial infarctions and non-fatal cerebral strokes, by $14 \%$. Furthermore, treatment using empagliflozin resulted in lower death rates from any cause (32\% reduction) and fewer hospitalizations for heart failure (35\% reduction) [25].

The most common side effects connected with this drug class stem from the basic mechanism of their action: glycosuria promotes more frequent infections of the genitourinary system, particularly infections of fungal etiology. Since SGLT2 inhibitors constitute a new drug class, the long-term safety of their administration requires further research and observations. There is an increasing number of reports of increased risk of ketoacidosis or severe urinary tract infections, including urosepsis [26-29].

\section{SGLT2 inhibitors and ketoacidosis}

On 15 May 2015 the FDA in the US, whose purpose is to control and approve drugs for marketing, issued, based on an analysis of the FDA Adverse Event Reporting System (FAERS) database, a communication warning about the increased risk of ketoacidosis with low or mild increase in blood glucose concentration (euglycemic diabetic ketoacidosis - euDKA) connected with taking the SGLT2 inhibitors registered so far. Between March 2013 to 6 June 2014, 20 cases of ketoacidosis or ketosis were identified in patients with diabetes treated using this drug class. All cases required hospitalization. They were mostly patients with type 2 diabetes; a few cases concerned patients with type 1 diabetes. In several reports, the type of diabetes the person taking the SGLT2 inhibitor had was not specified [27].

A month later, EMA in Europe informed that, until 19 May 2015, 147 cases of ketoacidosis in patients with diabetes treated using SGLT2 inhibitors were registered in the EudraVigilance database [28]. 101 cases concerned type 2 diabetes, while 46 cases were reported in patients with type 1 diabetes who used the SGLT2 inhibitor "off-label" (Tab. 1).

Of these 147 people with reported cases of ketoacidosis, 96 used canagliflozin, 46 used dapagliflozin and 5 used empagliflozin. All the cases were serious and 69 required hospitalization. Time to onset of ketoacidosis following inclusion of gliflozin varied between 3 days to 1 year. Most episodes occurred during the first two months of treatment.
In another notice, issued 4 December 2015, the FDA recommended adding warnings about increased risk of ketoacidosis and serious urinary tract infections while taking this class of drugs to labels of SGLT2 inhibitors [27]. Simultaneously, it was announced that, on the basis of an analysis of FAERS reports, between March 2013 (approval of first gliflozin) and May 2015 73 cases of ketoacidosis were identified in patients taking SGLT2 inhibitors. 48 patients used canagliflozin, 21 used dapagliflozin and 4 used empagliflozin. In all cases patients were either hospitalized or had to stay in the hospital's emergency department.

Of these 73 cases, 44 reports concerned type 2 diabetes, while 15 reports concerned patients with type 1 diabetes who used the SGLT2 inhibitor "off-label". In 13 cases the type of diabetes was not specified, however 10 patients were being treated concomitantly with other oral antidiabetic drugs, suggesting that they had type 2 diabetes as well. One case was reported in a patient with a previously undiagnosed LADA type diabetes. Of the reports concerning cases with type 2 diabetes, 34 contained information about possible combination treatment with insulin. Concurrent insulin therapy was used for 16 patients. Time to onset of ketoacidosis following inclusion or an increase in the dose of gliflozin varied between 1 day to 1 year (43 days on average). Though considering the number of people taking SGLT2 inhibitors (over five hundred thousand patients) the number of described complications seems small, it was noted that mildly increased blood glucose levels combined with developing acidosis may cause a delay in diagnosis, and as a consequence a significant increase in ketoacidosis.

At the same time, it was announced that 19 cases of urosepsis in patients with diabetes treated using SGLT2 inhibitors had been registered in the FAERS database between March 2013 and October 2014. Canagliflozin was used by 10 people, while dapagliflozin by 9 . No deaths were reported. 4 patients had to be admitted to an intensive care unit and 3 had to be dialysed due to renal insufficiency. The average length of time the drug was being used prior to the onset of the infection was 45 days (between 2 and 270 days). In 15 cases, further administration of SGLT2 was discontinued.

\section{Diabetic ketoacidosis without hyperglycemia Clinical picture}

Peters et al. presented cases of diabetic ketoacidosis without hyperglycemia in 9 patients, including 7 with type 1 diabetes and 2 with type 2 diabetes, who were taking an SGLT2 inhibitor [30]. All patients 
Table 1. Cases of ketoacidosis in patients with diabetes taking SGLT2 inhibitors, registered in the EudraVigilance database until 19 May 2015

\begin{tabular}{lccc}
\hline $\begin{array}{l}\text { Name } \\
\text { of drug }\end{array}$ & $\begin{array}{c}\text { Patients } \\
\text { with type 2 } \\
\text { diabetes }\end{array}$ & $\begin{array}{c}\text { Patients } \\
\text { with type 1 } \\
\text { diabetes }\end{array}$ & $\begin{array}{c}\text { Cases reported } \\
\text { in the European } \\
\text { Union }\end{array}$ \\
\hline Dapagliflozin & 34 & 12 & 18 \\
Canagliflozin & 63 & 33 & 0 \\
Empagliflozin & 4 & 1 & 0 \\
\hline
\end{tabular}

were being treated with canagliflozin, which is most likely due to the fact that this drug was the first to be introduced in the United States and saw the most widespread use. No patient had a previous episode of diabetic ketoacidosis. Nearly all reported nausea and vomiting. It would seem, however, that nausea was a consequence of rather than a factor causing acidosis. Stomach aches, headaches and dizziness were observed in singular cases. Tachypnea and tachycardia were observed in one (female) patient who had no gastrointestinal symptoms.

In patients with type 1 diabetes, ketoacidosis was preceded by infection, consumption of alcohol, increased physical activity and/or decreased intake of carbohydrates, often in conjunction with a decrease in the dose of insulin or discontinuation of its use. In patients with type 2 diabetes, ketoacidosis occurred soon after surgery.

The patients described had normal or slightly elevated blood glucose levels, which resulted in a significant delay in diagnosis of ketoacidosis and consequently in administration of appropriate treatment (Tab. 2). However, once the diagnosis was made, the patients' condition rapidly improved after including liquids and insulin, administered intravenously. In several cases, a recurrence of ketoacidosis was observed after resuming administration of the SGLT2 inhibitor.

\section{Pathophysiology}

Diabetic ketoacidosis is a complex disorder in carbohydrate, fat and protein metabolism as well as water-electrolyte and acid-base balance, stemming from profound insulin deficiency. It is a severe metabolic complication which may occur during the course of any type of diabetes. It typically happens in patients with type 1 diabetes, less often in those with type 2 diabetes.

Diabetic ketoacidosis is a consequence of a significant deficiency or lack of insulin accompanied by an increased concentration of insulin-counteracting hormones, such as glucagon, cortisol, catecholamines or growth hormone. Insulin deficiency, through decreased
Table 2. Results of laboratory tests of patients taking an SGLT2 inhibitor in whom ketoacidosis without hyperglycemia was diagnosed, as described by Peters et al.

\begin{tabular}{lcc}
\hline Parameters tested & $\begin{array}{c}\text { Patients with } \\
\text { type 2 } \\
\text { diabetes } \\
(\mathbf{n}=\mathbf{2})\end{array}$ & $\begin{array}{c}\text { Patients with } \\
\text { type 1 } \\
\text { diabetes } \\
(\mathbf{n}=\mathbf{7})\end{array}$ \\
\hline Glycemia [mg/dL] & $150-169$ & $96-233$ \\
$\mathrm{pH}$ & 7.12 & $6.9-7.15$ \\
Anion gap [mEq/L] & $16-19$ & $17-35$ \\
Bicarbonates [mmol/L] & $5-10$ & $6-18$ \\
Ketones in blood or urine & High & High \\
& concentration & concentration \\
\hline
\end{tabular}

glucose uptake in insulin-dependent tissues and increased production of glucose via gluconeogenesis and glycogenolysis, leads to hyperglycemia. Lipolysis results in an excess of free fatty acids and glycerol. Free fatty acids undergo re-esterification into triglycerides and $\beta$-oxidation, which leads to increased ketogenesis in the liver. Overproduction of ketone bodies causes development of metabolic acidosis. Catabolic state is complemented by increased proteolysis. Catabolism of proteins leads to the creation of amino acids, which provide substrate for glucose production (gluconeogenesis). Hyperglycemia and ketonemia activate the mechanisms of osmotic diuresis. Loss of water leads to dehydration and electrolyte disorders. This results in hyperosmolarity of the blood plasma and impairs blood perfusion in organs. Free water and electrolyte deficit, augmented by vomiting and rapid breathing as well as increasing acid-base imbalance, leads to disruptions in the functioning of the central nervous system and coma.

Laboratory diagnostic criteria for diabetic ketoacidosis include: hiperglycemia > $250 \mathrm{mg} / \mathrm{dl}$ ( $13.9 \mathrm{mmol} / \mathrm{L}$ ), though the values are usually much higher, up to 600 $-800 \mathrm{mg} / \mathrm{dL}$ or more; $\mathrm{pH}$ of arterial or capillary blood $<7.3$; bicarbonate serum concentration < $15 \mathrm{mmol} / \mathrm{L}$; presence of ketone bodies in urine or serum; increased anion gap > $12 \mathrm{mmol} / \mathrm{L}$ [15].

Diabetic ketoacidosis does not always cause increased blood glucose levels. In 1973 Munro et al. presented 211 cases of ketoacidosis, of which 37 were described as euglycemic ketoacidosis, defined as blood glucose concentration $<300 \mathrm{mg} / \mathrm{dL}(16.7 \mathrm{mmol} / \mathrm{L})$ and bicarbonate serum concentration $\leq 10 \mathrm{mEq} / \mathrm{L}$ [31]. These cases concerned young people with type 1 diabetes, of which $2 / 3$ were women. The primary cause of acidosis developing in the patients was decreased carbohydrate intake due to vomiting or starvation, oc- 
casionally coupled with reduced insulin dose. In later years it was accepted that serum glucose concentration in euglycemic ketoacidosis is $\leq 200 \mathrm{mg} / \mathrm{dl}(11,1 \mathrm{mmol} / \mathrm{l})$ $[32,33]$. However, it is not a value which could be defined as normoglycemia. Hence the name "ketoacidosis without hyperglycemia" seems more appropriate.

The fact that cases of ketoacidosis without hyperglycemia while taking SGLT2 inhibitors were reported mostly in patients with prolonged diabetes, including those treated using insulin and those with type 1 diabetes, indicates that insulin deficiency is an important pathogenic factor in this disorder. At the same time, in most cases there was a decrease in the insulin dose administered so far due to low blood glucose levels. In some patients, a significant reduction in blood glucose levels was likely only the effect of increased urinary glucose excretion promoted by SGLT2 inhibitors. In addition, in many of them blood glucose levels decreased also as a result of reduced appetite, nausea, sometimes vomiting or refraining from eating due to surgery. Consumption of alcohol on the day preceding the onset of the ailment may have also been one of the causes of reduced blood glucose levels. Regardless of reason, lower blood glucose levels led to a decrease in the administered insulin doses, and in case of type 2 diabetes to reduced secretion of endogenous insulin.

Urinary glucose excretion while taking SGLT2 inhibitors is approx. 50-100 g/day. In overweight or obese patients, who consume $50 \%$ of their daily requirement as carbohydrates, such level of excretion constitutes approx. 17-34\% of carbohydrate intake for men and $22-44 \%$ for women. In people who are not significantly overweight, urinary glucose excretion equals approx. $50 \%$ of daily carbohydrate intake, for both men and women [34]. The amount of glucose available for metabolic processes in tissues is reduced, especially since there is a concurrent decrease in circulating endogenous insulin or exogenous insulin dosage. Lower insulin concentration leads to a significant increase in glucagon concentration in the serum. This stems from the fact that insulin's inhibitory, paracrine effect on $\alpha$-cells is diminished, and possibly also from reduced glucose transport to these cells due to SGLT2 inhibition $[35,36]$.

Lower blood glucose levels and insulin concentration with a simultaneous increase in glucagon levels lead to reduced glucose influx into cells, reduced glycolysis and increased lipolysis and $\beta$-oxidation of free fatty acids. This, in turn, causes an increased rate of synthesis of ketone bodies in the liver. The increase in glucagon concentration may also be stimulated through hypovolemia and orthostatic hypotension, which may happen during the first three months of
SGLT2 treatment and are a result of increased diuresis and consequently hypovolemia. Concentrations of cortisol and catecholamines, which also take part in intensifying lipolysis processes (Fig. 1), may also rise at this time for the same reasons.

Perhaps in some cases the described sequence of metabolic disorders is sufficient for minor ketoacidosis to occur; it exhibits such symptoms as malaise, reduced appetite, nausea, vomiting or headache. Since at the same time blood glucose levels remain relatively low, patients do not suspect the possibility of this complication and decide to decrease or skip their insulin dose. The result is further increase in acidosis and severe deterioration of the patient's state of health.

In some patients, described metabolic disorders may be caused by a situation leading to limited meal consumption, including carbohydrates, e.g. dieting, starvation, preparing for a medical examination, stomach aches, surgery or other, sometimes insignificant disorders causing reduced appetite. The amount of available glucose, already lowered through glycosuria caused by SGLT2 inhibitors, is further reduced, causing the whole sequence of disorders described above. In this case it will also ultimately lead to ketoacidosis with normoglycemia or slight hyperglycemia.

\section{Safety and prevention methods}

On 16 February 2016, the Pharmacovigilance Risk Assessment Committee (PRAC) of the European Medicines Agency published recommendations meant to minimize the risk of ketoacidosis in patients undergoing treatment using SGLT2 inhibitors [37]. Patients taking any such drugs should be aware of the symptoms of ketoacidosis, including body weight loss, nausea or vomiting, stomach aches, excessive thirst, rapid and deep breathing, confusion, unusual sleepiness or fatigue, sweet-smelling breath, sweet or metallic taste in the mouth or change in urine or sweat odour. It is worth to inform the patient about the need to monitor ketonuria and/or ketonemia, despite "good" blood glucose levels, in situations promoting ketogenesis: during another disease, during periods of reduced food and fluid intake, after reducing insulin doses, if nausea and/ /or vomiting occur, after alcohol consumption. Furthermore, PRAC recommends to temporarily cease SGLT2 inhibitor treatment in patients during perioperative period or hospitalized due to severe illness.

\section{Summary}

Available hypoglycemic drugs often do not make it possible to attain therapy goals or their effectiveness is reduced due to side effects. Currently, research to find new therapy options with a different mechanism 


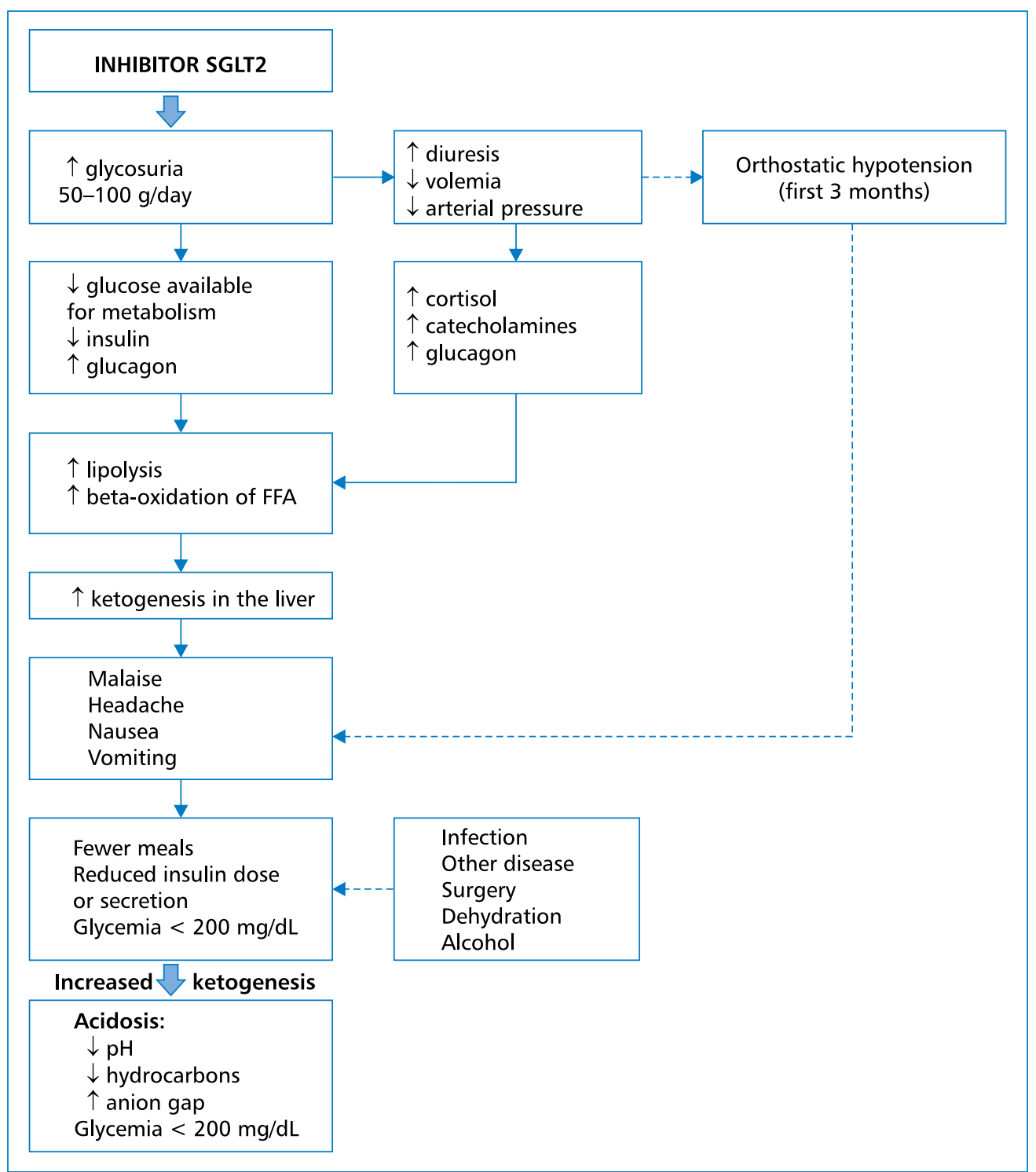

Figure 1. Possible pathogenic mechanisms of ketoacidosis without hyperglycemia while taking SGLT2 inhibitors

of action is being conducted on a large scale. SGLT2 inhibitors (gliflozins), which impede renal glucose reabsorption, constitute a new drug class. Their mechanism of action is not dependent on $\beta$ cells functioning or insulin sensitivity. They improve the control of blood glucose levels in patients with type 2 diabetes, both when used as monotherapy as well as in combination therapy with other hypoglycemic drugs or insulin.

Gliflozins have been available on the market for 4 years. Databases of adverse effects indicate the possibility of diabetic ketoacidosis in patients taking them. An unusual course of this type of ketoacidosis, with normal or relatively low glucose concentration, makes it potentially difficult to diagnose. Though the risk of this type of ketoacidosis occurring in patients with type 2 diabetes while taking SGLT2 inhibitors seems very low, it is nevertheless worth to be aware of such a complication. The risk may increase during periods of reduced meal consumption, starvation (e.g. in perioperative period), other diseases which increase the need for insulin, stress or in patients with prolonged diabetes, in whom the dysfunction of $\beta$ cells is greater.

Should symptoms which may conform to ketoacidosis occur, the basic diagnostic method, possible to perform even at home, ought to be testing for ketone bodies in urine or in blood. Such a test should be performed even if there is no significant hyperglycemia. The presence of ketones should be a signal to ingest carbohydrates, apply insulin and increase the amount of fluids taken, while simultaneously ceasing to take the drug. If the symptoms are mild, such action may be sufficient to correct metabolic disorders and halt the 
ailment. General malaise and exacerbation of symptoms indicate a need for hospital treatment. It is important, in case clinical symptoms conforming to ketoacidosis occur in patients taking SGLT2 inhibitors, regardless of type of diabetes, to remember about the possibility of this complication, even if blood glucose levels are within normal range or only slightly elevated. Laboratory diagnosis is simple, as is treatment, if applied in time.

If diabetic ketoacidosis is suspected or confirmed, gliflozin treatment should be ceased immediately and not resumed, unless a different cause of acidosis is determined.

The safety of long-term administration of drugs from this class requires further research.

\section{REFERENCES}

1. International Diabetes Federation. Diabetes Atlas. Seventh Edition [online]. International Diabetes Federation, (C) 2015. [accessed 14 February 2016]. Available at: http://www.diabetesatlas.org.

2. McEwen LN, Karter AJ, Curb JD et al. Temporal trends in recording of diabetes on death certificates: results from Translating Research Into Action for Diabetes (TRIAD). Diabetes Care 2011; 34: 1529-1533.

3. World Health Organization. Global Health Observatory Data Repository 2013.

4. Gale EA. Is there really an epidemic of type 2 diabetes. Lancet 2003; 362: 503-504.

5. Wright EM, Hirayama BA, Loo DF. Active sugar transport in health and disease. J Intern Med 2007; 261: 32-43.

6. Rahmoune H, Thompson PW, Ward JM et al. Glucose transporters in human renal proximal tubular cells isolated from the urine of patients with non-insulin-dependent diabetes. Diabetes 2005; 54: 3427-3434.

7. DeFronzo RA. From the triumvirate to the ominous octet: a new paradigm for the treatment of type 2 diabetes mellitus. Diabetes 2009; 58: 773-795.

8. Ehrenkranz JR, Lewis NG, Kahn CR et al. Phlorizin: a review. Diabetes Metab Res Rev 2005; 21: 31-38.

9. European Medicines Agency. Human Medicines. Forxiga dapagliflozin [online]. EMA, (C1995-2016. [accessed 14 February 2016]. Available at: http://www.ema.europa.eu/ema/index. jsp?curl=pages/medicines/human/medicines/002322/human_ med 001546.jsp\&mid=WC0b01ac058001d124.

10. European Medicines Agency. Human Medicines. Invokana canagliflozin[online]. EMA, (C1995-2016. [accessed 14 February 2016]. Available at: http://www.ema.europa.eu/ema/index. jsp?curl=pages/medicines/human/medicines/002649/human_ med 001707.jsp\&mid=WCOb01ac058001d124.

11. European Medicines Agency. Human Medicines. Jardiance empagliflozin [online]. EMA, (C)1995-2016. [accessed $14 \mathrm{Fe}-$ bruary 2016]. Available at: http://www.ema.europa.eu/ema/ /index.jsp?curl=pages/medicines/human/medicines/002677/ /human med 001764.jsp\&mid=WC0b01ac058001d124.

12. U.S. Food and Drug Administration. FDA news release. FDA approves Invokana to treat type 2 diabetes [online]. [accessed 14 February 2016]. Available at: http://www.fda.gov/NewsEvents/ Newsroom/PressAnnouncements/ucm345848.htm

13. U.S. Food and Drug Administration. FDA news release. FDA approves Farxiga to treat type 2 diabetes [online]. [accessed 14 February 2016]. Available at: http://www.fda.gov/newsevents/ newsroom/pressannouncements/ucm380829.htm

14. U.S. Food and Drug Administration. FDA news release. FDA approves Jardiance to treat type 2 diabetes [online]. [accessed 14 February 2016]. Available at: http://www.fda.gov/NewsEvents/ /Newsroom/PressAnnouncements/ucm407637.htm.

15. Zalecenia kliniczne dotyczące postępowania u chorych na cukrzycę 2016. Stanowisko Polskiego Towarzystwa Diabetologicznego. Diabetologia Kliniczna 2016; 5 (supl. A): A1-A76.

16. Ferrannini E, Solini A. SGLT2 inhibition in diabetes mellitus: Rationale and clinical prospects. Nat Rev Endocrinol 2012; 8: 495-502.
17. Stenlöf K, Cefalu WT, Kim KA et al. Efficacy and safety of canagliflozin monotherapy in subjects with type 2 diabetes mellitus inadequately controlled with diet and exercise. Diabetes Obes Metab 2013; 15: 372-382.

18. Liang Y, Arakawa K, Ueta K et al. Effect of canagliflozin on renal threshold for glucose, glycemia, and body weight in normal and diabetic animal models. PLoS One 2012; 7: e30555.

19. Sabino-Silva R, Mori RC, David-Silva A et al. The Na+/glucose cotransporters: from genes to therapy. Braz J Med Biol Res 2010; 43: 1019-1026.

20. Liu JJ, Lee T, DeFronzo RA. Why do SGLT2 inhibitors inhibit only $30-50 \%$ of renal glucose reabsorption in humans? Diabetes 2012; 61: 2199-2204.

21. Tikkanen I, Narko K, Zeller $C$ et al. EMPA-REG BP Investigators. Empagliflozin reduces blood pressure in patients with type 2 diabetes and hypertension. Diabetes Care 2015; 38: 420-428.

22. Majewski C, Bakris GL. Blood pressure reduction: an added benefit of sodium-glucose cotransporter 2 inhibitors in patients with type 2 diabetes. Diabetes Care 2015; 38: 429-430.

23. Clar C, Gill JA, Court R et al. Systematic review of SGLT2 receptor inhibitors in dual or triple therapy in type 2 diabetes. BMJ [online]. 2012; 2: e001007. [accessed 14 February 2016]. Available at: doi:10.1136/bmjopen-2012-001007.

24. Monami M, Nardini C, Mannucci E. Efficacy and safety of sodium glucose co-transport- 2 inhibitors in type 2 diabetes: $A$ meta-analysis of randomized clinical trials. Diabetes Obes Metab 2014; 16: 457-66.

25. Zinman B, Wanner C, Lachin JM et al. Empagliflozin, cardiovascular outcomes, and mortality in type 2 diabetes. $N$ Engl J Med [online]. 2015; 373: 2117-2128. [accessed 14 February 2016]. Available at: doi: 10.1056/NEJMoa1504720.

26. U.S. Food and Drug Administration. FDA Drug Safety Communication: FDA warns that SGLT2 inhibitors for diabetes may result in a serious condition of too much acid in the blood [online]. [accessed 14 February 2016]. Available at: http://www.fda.gov/ /drugs/drugsafety/ucm446845.htm.

27. U.S. Food and Drug Administration. FDA Drug Safety Communication. SGLT2 inhibitors: drug safety communication - labels to include warnings about too much acid in the blood and serious urinary tract infections [online]. [accessed 19 February 2016]. Available at: http://www.fda.gov/Drugs/DrugSafety/ucm475463.htm.

28. European Medicines Agency. Human Medicines. Notification to the $P R C / E M A$ secretariat ofa referral under article 20 of regulation (EC) 726/2004 [online]. EMA, (C1995-2016. [accessed 10 February 2016]. Available at: http://www.ema.europa.eu/docs/en_GB/ document_library/Referrals_document/SGLT2_inhibitors_20/ Procedure_started/WC500187925.pdf.

29. European Medicines Agency. Human Medicines. Review of diabetes medicines called SGLT2 inhibitors started: risk of diabetic ketoacidosis to be examined [online]. EMA, (1)1995-2016. [accessed 14 February 2016]. Available at: http://www.ema.europa.eu/ /docs/en_GB/document_library/Referrals_document/SGLT2_inhibitors 20/Procedure started/WC500187926.pdf.

30. Peters AL, Buschur EO, Buse JB et al. Euglycemic diabetic ketoacidosis: a potential complication of treatment with sodium-glucose cotransporter 2 inhibition. Diabetes Care 2015; 38: 1687.

31. Munro JF, Campbell IW, McCuish AC et al. Euglycaemic diabetic ketoacidosis. Br Med J 1973; 2: 578-580.

32. McNulty SJ, English PJ. Euglycaemic diabetic ketoacidosis. Practical Diabetes International. 2002; 19: 63.

33. Davies RG, De P, Child DF et al. Euglycaemic diabetic ketoacidosis. Hospital Medicine. 2003; 64: 557-558.

34. Rosenstock J, Ferrannini E. Euglycemic diabetic ketoacidosis: a predictable, detectable, and preventable safety concern with SGLT2 inhibitors. Diabetes Care 2015; 38: 1638-1642.

35. Maruyama $H$, Hisatomi A, Orci $L$ et al. Insulin within islets is a physiologic glucagon release inhibitor. J Clin Invest 1984; 74: 2296-2299.

36. Bonner C, Kerr-Conte J, Gmyr V et al. Inhibition of the glucose transporter SGLT2 with dapagliflozin in pancreatic alpha cells triggers glucagon secretion. Nat Med 2015; 21: 512-517.

37. European Medicines Agency. Human Medicines. SGLT2 inhibitors: PRAC makes recommendations to minimise risk of diabetic ketoacidosis [online]. EMA, (C)1995-2016. [accessed 21 February 2016]. Available at: http://www.ema.europa.eu/ema/index. jsp?curl=pages/news_and_events/news/2016/02/news_detail 002470 .jsp\&mid=WC0b01ac058004d5c1. 